\title{
Intermittency in local field potentials recorded from the thalamus of patients with essential tremor Asok Sen*1, Abdoul Kane ${ }^{2}$, William D Hutchison²,3, Andres M Lozano ${ }^{3}$, Mojgan Hodaie ${ }^{3}$ and Jonathan O Dostrovsky ${ }^{2}$
}

\author{
Address: ${ }^{1}$ Department of Mathematical Sciences, Indiana University, 402 N. Blackford Street, Indianapolis, IN 46202, USA, ${ }^{2}$ Department of \\ Physiology, University of Toronto, 1 King's College Circle, Toronto, ON M5S 1A8, Canada and ${ }^{3}$ Department of Surgery, University of Toronto, \\ 100 College Street, Toronto, ON M5G 1L5, Canada \\ Email: Asok Sen* - asen@iupui.edu \\ * Corresponding author
}

from Sixteenth Annual Computational Neuroscience Meeting: CNS*2007

Toronto, Canada. 7-12 July 2007

Published: 6 July 2007

BMC Neuroscience 2007, 8(Suppl 2):PI4I doi:I0.I I86/I47I-2202-8-S2-PI4I

() 2007 Sen et al; licensee BioMed Central Ltd.

We have recorded the local field potentials (LFPs) from 12 sites in the motor thalamus of 4 patients with essential tremor who were undergoing surgery for implantation of deep brain stimulation (DBS) electrodes for the treatment of their tremor. By band-pass filtering the LFPs (20 - 40 seconds of recording at each site) and applying a continuous wavelet transform on the filtered signals, we detected the presence of marked intermittency in the theta frequency band $(4-8 \mathrm{~Hz})$ at many of these sites (see Fig. 1). We have also computed the temporal variations of wavelet power to demonstrate that the intermittency is characterized by sudden bursts of power separated by intervals of very low power or almost quiescent periods. In addition, the kurtosis of the probability density functions of the LFP signals was used as a measure of the degree of intermittency at each site and revealed a wide variation ranging from 3.4 to 37.5 . The larger the kurtosis, the greater is the intermittency in the signal. The origin and significance of the intermittency are presently unclear. It has been postulated that the cerebello-thalamo-cortical loop may be responsible for the generation of $6-9 \mathrm{~Hz}$ oscillatory electromyographic activity observed during voluntary movements, and possibly the bursts of theta oscillatory activity observed in the thalamus of these patients may be related to fluctuations of activity in this circuit.
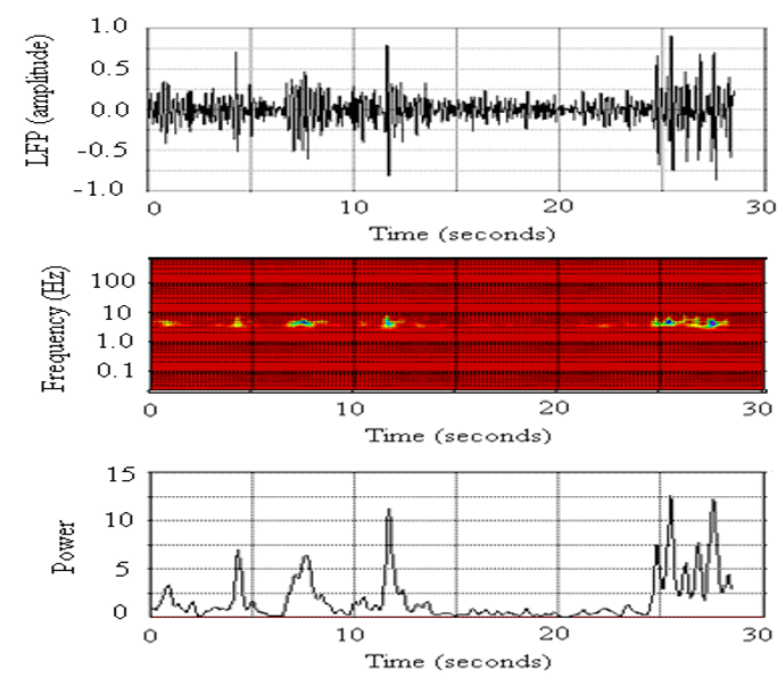

Figure I

Wavelet analysis of the LFP signal from the thalamus of a patient with essential tremor. Upper panel - Time series of the LFP signal; Middle panel - Wavelet power spectrum; Lower panel - Temporal variation of wavelet power; For this signal, the kurtosis is equal to 6.98 . 